\title{
INMIGRACIÓN, CRISIS Y ESCUELA ${ }^{1}$ IMMIGRATION, CRISIS AND SCHOOL
}

F. Javier García Castaño, Antonia Olmos Alcaraz y OuafaA Bouachra Outmani*

Resumen: Es fácil encontrar titulares de prensa en España en los últimos años que digan: "la crisis ha destruido empleo y la falta del mismo ha obligado a los inmigrantes a retornar a sus países...". A esos titulares se añaden afirmaciones como "ya no llegan inmigrantes...». Ello ha servido para justificar la reducción de los servicios que se les podía prestar. También en la escuela se escuchan voces similares y con ello la reducción de apoyos educativos con el argumento de que "ya no llegan» o "se han marchado». Pues bien, lo que pretendemos con este trabajo es poner a prueba ese conjunto de afirmaciones que en la escuela relacionan "crisis económica»y "retorno" de la población de nacionalidad extranjera. Tres puntos trataremos con detalle: en primer lugar analizaremos cómo ha evolucionado la presencia de escolares de nacionalidad extranjera en el sistema educativo en España, en segundo lugar nos serviremos de la Estadística de Migraciones y del Padrón Municipal de Habitantes para conocer mejor el fenómeno migratorio y sus asociados: el "retorno", y en tercer lugar reflexionaremos sobre cómo se está utilizando la idea del "retorno» la población inmigrante para reducir los servicios que se les presta en el sistema educativo.

* Instituto de Migraciones, Universidad de Granada

1 Este texto es deudor del Proyecto del Plan Nacional de I+D+i: Construyendo diferencias en la escuela. estudios de las trayectorias de las ATAL en Andalucía, de sus profesorado y de su alumnado (CSO2013-43266-R). Agradecemos a dicho Plan Nacional la financiación para el desarrollo del mismo. Queremos reconocer también los comentarios a una primera versión de este texto de Borja Fernández y Rocío García. Nuestros más sincero agradecimiento a los comentarios críticos de Andreu Domingo. 
Palabras clave: inmigración, crisis, retorno, escuela, España

Abstract: In recent years, it is common to find headlines in the Spanish Press saying, "employment has dwindled due to the crisis and the lack of jobs has forced immigrants to return to their natives homes..." Added to these headlines, assertions such as "immigrants are no longer coming" can be found. These statements have been used to justify the gradual reduction of the services provided to this population. At the school level, similar voices are being heard, supporting the reduction of educationalaids alleging that «immigrants are not coming" or "they have left». In this paper, we have attempted to evaluate this set of allegations that relate the "economic crisis" to "the return" of the population of foreign nationality. For that purpose, three major issues will be addressed. Firstly, the evolution of the foreign population attending the Spanish school system will be analysed. Secondly, the Statistics on Migrations and the Municipal Register of Inhabitants will be used to examine the issues related to the immigrants' return. Finally, we will reflect on how the notion of "return" of the foreign population is being used in order to reduce the resources that are provided to them in the educational system.

Keywords: immigration, crisis, return, school, Spain

De entre los muchos fenómenos sociales que se han visto afectados por la llamada "crisis económica», se encuentra también el fenómeno migratorio. La ecuación parece sencilla: «la crisis ha destruido empleo y la falta del mismo ha obligado a los inmigrantes a retornar a sus países...». Los propios medios de comunicación se han apuntado a esta relación entre «crisis» $\mathrm{y}$ «retorno». En este sentido, leíamos en el periódico El País (13/08/2013) «La recesión ha empujado a muchos inmigrantes que llegaron a Cataluña en tiempos de bonanza a regresar a su país» ${ }^{2}$. Y unos meses antes ya habíamos escuchado en los informativos de Radio Televisión Española un reportaje en el mismo sentido: «El éxodo de los inmigrantes que retornan a sus países por la crisis económica ha provocado que la población española baje por primera vez desde $1996 \ldots »^{3}$.

2 http://ccaa.elpais.com/ccaa/2013/08/12/catalunya/1376338721_379266.html

3 http://www.rtve.es/noticias/20130422/poblacion-espanola-baja-primeravez-desde-1996-regreso-inmigrantes-paises/646320.shtml 
Algunas personas no se han limitado a proponer visiones simplistas, sino que han propiciado tal simplicidad, a pesar de que los expertos en la materia han advertido que medir los flujos de retorno no es tarea fácil si tenemos en cuenta las actuales estadísticas de las que disponemos en España. López de Lera (2010: 16) nos indica que la «medición» del retorno plantea problemas analíticos (de naturaleza, de medición, de compatibilidad y de cobertura) y Andreu Domingo viene insistiendo sobre estas cuestiones:

... el sistema estadístico español tiene graves problemas para captar las bajas padronales que se dirigen al extranjero, sean éstas movimientos de retorno (el inmigrado que vuelve a su país de origen), movimientos de arrastre (la persona nacida en España, con o sin la nacionalidad española, relacionada con un inmigrante que se trasladará al país de origen de este), o los movimientos a terceros países (...) de la Unión Europea (Domingo y Recaño, 2010: 186).

Sin embargo, además de las necesarias prudencias de las que nos advierten esas palabras, existen estudios que relativizan la relación causa-efecto entre «crisis económica» y «retorno». Meses antes de aquellas noticias que relacionaban "crisis» y "retorno», los expertos en estas cuestiones trataban de explicar que ni los datos de retornos son tan importantes, ni todos los datos de «pérdidas» de población deben ser vistos como retornos:

A efectos de padrón, los nacionalizados cuentan como españoles. Son, sobre todo en el caso de los latinos, «una transfusión sanguínea a la cifra de españoles», según el demógrafo Izquierdo. «La pequeña variación del número de extranjeros no quiere decir que se estén yendo. Se puede explicar con creces con las naturalizaciones», afirma Arango (El País, 18/01/2013).

Pero no son sólo opiniones en la prensa. Los estudios también ponen en entredicho la idea de un "retorno masivo» de inmigrantes. Aunque es cierto que el saldo de población inmigrante se ha reducido de 900.000 en 2007 a 100.000 en 2011, el descenso se ha debido sobre todo al colapso en las nuevas entradas (Colectivo Ioe, 2012). Estaríamos más ante una ilusión de «saldo negativo» que ante una realidad efectiva de retorno. Según el INE, no es hasta 2011 cuando 
se producen efectivamente más salidas que entradas, ampliándose incluso en 2012 (Ibidem). Y es que la intención al hablar de «retorno masivo» puede ir en otra dirección si se potencia la idea de «salida masiva»: estamos contribuyendo a la creación de una cortina de humo que podría estar justificando la reducción de derechos de los extranjeros (Ibidem) y, así mismo, la disminución de políticas de atención a la diversidad ${ }^{4}$.

Un ejemplo destacado de esta conexión entre «crisis» y «retorno» se observa también en la escuela en relación con la población que allí se denomina inmigrante. Se han dado al menos dos explicaciones conectadas:

a. Afirmaciones tales como «ya no llegan inmigrantes» y «se están marchando muchos de los que llegaron $»^{5}$.

b. La constatación de que determinados apoyos educativos se han visto reducidos con el argumento de que «ya no llegan» 0 «se han marchado» ${ }^{6}$.

Pues bien, lo que pretendemos con este trabajo es poner a prueba ese conjunto de afirmaciones que en la escuela relacionan «crisis económica» $\mathrm{y}$ «retorno» de la población de nacionalidad extranjera. Tres puntos trataremos con detalle:

1. En primer lugar analizaremos cómo ha evolucionado la presencia de escolares de nacionalidad extranjera en el sistema

4 Esta relación entre «recortes» en políticas educativas y «retorno» ya se reflejó en la prensa: «Catalunya recorta las aulas de acogida por el descenso de inmigrantes» (La Vanguardia, 03/07/2013). (http://www.lavanguardia.com/vida/20100421/53912604882/catalunya-recorta-las-aulas-de-acogida-por-el-descenso-de-inmigrantes.html)

5 Nuestras investigaciones sobre las relaciones entre el fenómeno de la inmigración y sus efectos en el sistema educativo se inician en 1990: La escolarización de la población infantil inmigrante... (PB91-0740-C03-01), Inmigración, exclusión social e integración es España (SEC96-0796), Integración de escolares denominados «inmigrantes» (SEJ2007-67155/SOCI), Éxitos y fracasos escolares (CSO2010-22154-C03-01). Ver publicaciones en http://ldei.ugr.es/javiergarcia/

6 Para muestra véanse noticias como: «La llegada de la crisis económica marcó un cambio de tendencia en lo que había sido, durante prácticamente una década, un constante incremento del alumnado extranjero en Canarias» (El Día, 16/06/2014) (http://eldia.es/2014-06-16/canarias/2-centros-privados-escolarizanalumnado-extranjero.htm). O esta otra en dirección muy similar: «La crisis vacía las aulas de inmigrantes» (Diario de Sevilla, 12/11/2013) (http://www.diariodesevilla.es/article/sevilla/1643797/la/crisis/vacia/las/aulas/inmigrantes.html) 
educativo en España (Estadísticas de Educación del Ministerio de Educación).

2. En segundo lugar nos serviremos de la Estadística de Migraciones y del Padrón Municipal de Habitantes para conocer mejor el fenómeno migratorio y sus asociados: el «retorno».

3. En tercer y último lugar reflexionaremos sobre cómo se está utilizando la idea del «retorno» de los inmigrantes para reducir los servicios que se les presta.

\section{EVOLUCIÓN DE LA PRESENCIA DE POBLACIÓN EXTRANJERA EN EL SISTEMA EDUCATIVO EN ESPAÑA}

La población extranjera ha aumentado su presencia en la escuela en los últimos 15 años $^{7}$. Podemos ver la evolución (cursos 1999/00 a 2012/13) en el Gráfico 1:

7 Las Estadísticas de Educación son elaboradas por el Ministerio de Educación a partir de la información facilitada por las Comunidades Autónomas. Se trata de estadísticas tipo stock de población extranjera, no estadísticas de migraciones. Se elaboran a partir de los informes estadísticos de los centros escolares remitidos al inicio de curso. Este procedimiento plantea dos problemas:

- Los datos facilitados por los centros al principio de cada curso impide contabilizar el registro de inscripciones que se producen a lo largo del curso. Para el caso de la población extranjera este hecho es importante, dado que se trata de una población con un alto grado de movilidad.

- Los responsables de los registros de los centros son las autoridades educativas de cada centro. Aunque en los últimos años se ha avanzado mucho en estos registros en lo relativo a la identificación de las nacionalidades, no siempre ha sido así. El escolar debe aportar la documentación que atestigua su nacionalidad, pero no faltan lugares en los que se discute cuál puede ser la nacionalidad de un escolar nacido en España de padre y/o madre extranjera (ver García, Gijón, Sánchez y Bolívar, 2005). 
GRÁFICO 1

\section{EVOLUCIÓN DE LA POBLACIÓN DE NACIONALIDAD EXTRANJERA EN EL SISTEMA EDUCATIVO EN ESPAÑA}

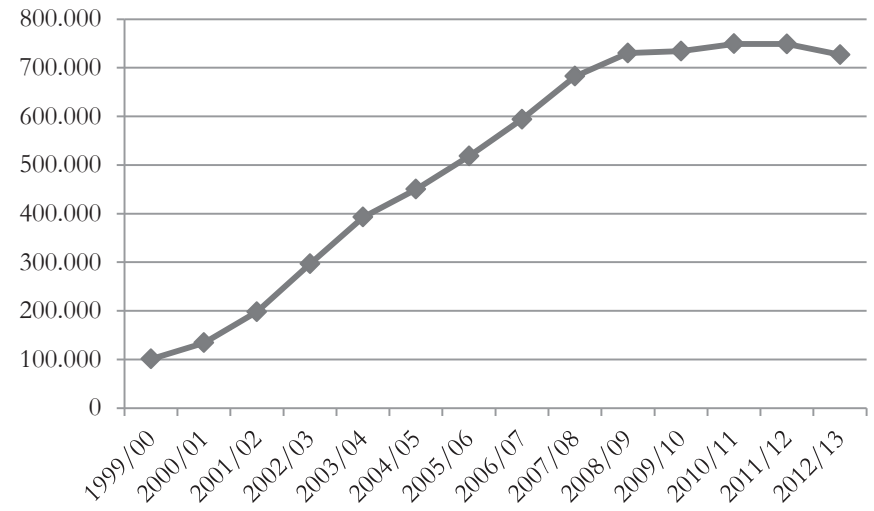

Fuente: elaboración propia a partir de Estadísticas de Educación ${ }^{8}$.

En la primera parte de la década del 2000 el crecimiento ha sido continuo, con tasas interanuales del $30 \%$ al $50 \%$; esos datos se moderan durante los años 2004-2007, quedándose en tasas del 15\%; y el cambio de década se caracteriza por una situación de incrementos muy ligeros (entre el 6\% y el 2\%). Tan sólo en los dos últimos cursos (2011/12 y 2012/13) se observa una disminución de esta población de menos del 1\% y el 3\% respectivamente. Ello indica que ahora «salen» más escolares de nacionalidad extranjera de los que «entran». Pero desagreguemos estos datos según tipo de enseñanza y nacionalidad. Nos centraremos en educación primaria y secundaria obligatoria, dado que son los dos únicos niveles plenamente obligatorios (ver Gráfico 2).

En el caso de la educación primaria observamos que se ha producido un crecimiento interanual del $30 \%$ al $50 \%$ al principio de la década, que se reduce en los siguientes años a menos del 15\%, para

8 http://www.mecd.gob.es/servicios-al-ciudadano-mecd/estadisticas/educacion/no-universitaria.html 
decrecer desde el curso 2009/10 y mantenerse así hasta el final de la serie (tasas desde el -4\% al -6\%). En el caso de la educación secundaria obligatoria los datos son muy similares: también crecimiento al inicio de la década, moderación a mitad, y decrecimiento más tarde (curso 2011/12, tasas del -2\% al -5\%). Estas dos enseñanzas son las únicas que presentan descensos. Ni en bachillerato, ni en educación infantil se observa lo mismo.

\section{GRÁFICO 2}

EVOLUCIÓN DE LA POBLACIÓN DE NACIONALIDAD EXTRANJERA EN EL SISTEMA EDUCATIVO EN ESPAÑA (EDUCACIÓN PRIMARIA Y SECUNDARIA OBLIGATORIA)

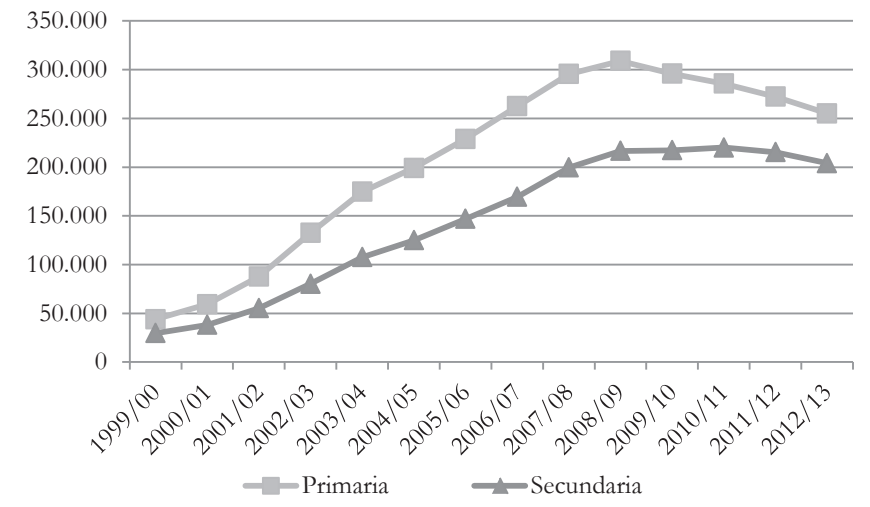

Fuente: elaboración propia a partir de Estadísticas de Educación. 
Observemos ahora los datos generales desagregados según nacionalidad. En el Gráfico 3 podemos ver cuatro de las nacionalidades de mayor importancia (cursos 1999/00 a 2012/13).

GRÁFICO 3

EVOLUCIÓN DE LA POBLACIÓN DE NACIONALIDAD EXTRANJERA EN EL SISTEMA EDUCATIVO EN ESPAÑA (CUATRO NACIONALIDADES)

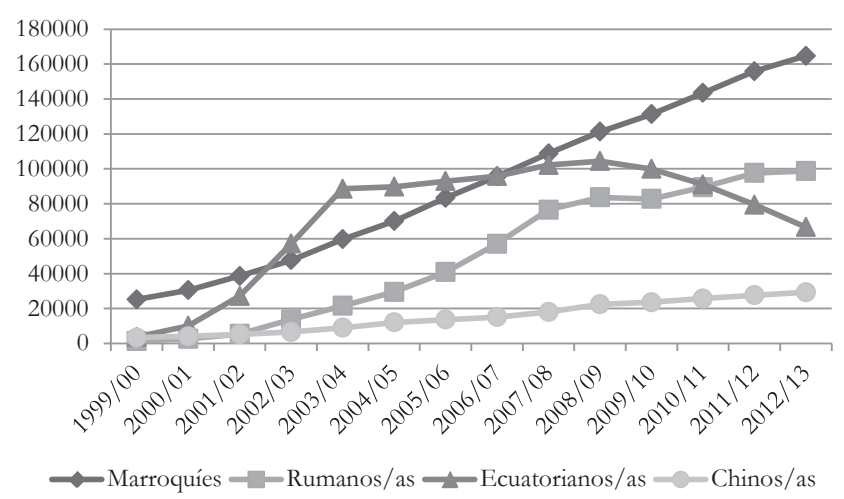

Fuente: elaboración propia a partir de Estadísticas de Educación.

En primer lugar tenemos a la población marroquí, cuyo peso ha ido oscilando entre el 23\% del principio de la serie al 21\% del final, aunque se trata de una población que en ningún momento ha parado de crecer. En segundo lugar tenemos a la población rumana que, aunque no siempre ha tenido una presencia muy significativa, se sitúa al final de la serie en la segunda población en importancia numérica (en 2012/13 el 13\%). Tampoco han dejado de crecer. En tercer lugar tenemos a la población ecuatoriana, que llegaron a colocarse como el grupo de mayor peso en los cursos 2002/03 a 2005/06, pero que desde entonces han visto como desciende su presencia absoluta y su peso relativo (son al final de la serie el $8 \%$ del total, pero llegaron a suponer hasta el 22\%). En cuarto lugar tenemos a la población china que, aunque no supone uno de los grupos más numeroso (3\%), tiene la misma tendencia que los dos primeros: crecimiento continuo. 
El conjunto de la población extranjera en el sistema educativo, por lo tanto, ha empezado a decrecer en el curso 2012/13, aunque en secundaria obligatoria tal decrecimiento ya empezó en el curso anterior y en primaria cuatro cursos antes. Tal descenso no se produce en otros niveles no obligatorios y se explica sólo por la disminución de la presencia de los nacionales de determinados países. En los ejemplos expuestos, de los más representativos, se observa que sólo la población ecuatoriana desciende. Detallemos algo más dicho descenso. En el conjunto de la enseñanza en el curso 2011/12 se escolarizaron 476 menores extranjeros menos, lo que suponía un decrecimiento interanual del -0,06\%. En el curso 2012/13 nos encontramos con 22.096 escolares menos, lo que supone un decrecimiento interanual del $-2,95 \%$.

No obstante, al estar contando la población extranjera debemos tener en cuenta que una parte de dicha población pueda nacionalizarse, con lo que de un curso para otro puede dejar de estar en la casilla de población de nacionalidad extranjera; ello, al menos, si las estadísticas se construyen de manera correcta (ver nota 8). Pues bien, a finales de 2012 el número de nacionalidades concedidas para población entre 0-14 años fue de 12.325 (Estadística Concesiones de nacionalidad española por residencia ${ }^{10}$ ), que muy bien podría ser una parte de los 22.096 extranjeros que en el curso 2012/13 habían «desaparecido» de las estadísticas del sistema educativo. El resultado supondría entonces una pérdida de 9.771 escolares, un 1,27\%.

Pasemos ahora a desagregar también los datos de decrecimiento según tipo de enseñanza y según nacionalidad. En el caso de la educación primaria la disminución comenzó en el curso 2009/10. En 2010/11 «desaparecen» de las estadísticas 10.249 escolares (un 3,46\%). En el curso siguiente la bajada es de 13.325 escolares $(4,67 \%)$. Y en el último curso analizado la reducción fue de 17.282 escolares $(6,35 \%)$. En tres cursos la educación primaria ha perdido a casi 15 escolares de nacionalidad extranjera de cada 100.

9 Cuando hablamos de «descenso» del conjunto de la población extranjera dicho cálculo es el resultado de sumar los nuevos escolares del sistema educativo y restar todos aquellos que lo abandonan.

10 Dicha estadística explota la base de datos gestionada por la Dirección General de los Registros y del Notariado del Ministerio de Justicia que registra todos los trámites relativos a la solicitud, concesión y denegación de la nacionalidad española por residencia. Web: http://extranjeros.empleo.gob.es/es/Estadisticas/operaciones/concesiones/index.html 
En el caso de la educación secundaria obligatoria los descensos se produjeron en los dos últimos cursos estudiados. El curso 2011/12 «desaparecen» de las estadísticas 4.666 escolares (respecto del año anterior, 2,12\% menos); y en el curso 2012/13 el descenso fue de 11.431 (un 5,31\% menos respecto al año anterior). En dos cursos la secundaria obligatoria ha "perdido» a poco más de 7 escolares de nacionalidad extranjera de cada 100 .

Estos mismos datos, analizados a la luz de la nacionalidad, nos ayudarán en nuestros argumentos. Nos centramos en las variaciones de los cursos los cursos 2011/12 y 2012/13. Las diferencias indican que 67 de las nacionalidades registradas en las estadísticas mostraban datos de descenso ${ }^{11}$. Otras 72 nacionalidades presentaban un aumento ${ }^{12}$. Del conjunto de nacionalidades que "perdían" alumnado hemos seleccionado las más importantes en relación con dicha "pérdida». Las siete más importantes suman el $80 \%$ del total del alumnado "perdido» y pertenecen a América del Sur. En la Tabla 1 podemos ver los datos:

\section{TABLA 1}

DIFERENCIA (Y PORCENTAJE SOBRE TOTAL DE «PÉRDIDAS») DE ALUMNADO EN EL SISTEMA EDUCATIVO EN ESPAÑA ENTRE EL CURSO 20011/12 Y EL 20012/13 DE LAS SEIS NACIONALIDAD CON MAYOR «PÉRDIDA» DE ALUMNADO

\begin{tabular}{|l|c|c|}
\hline & Diferencia & Porcentaje sobre el total de países \\
\hline Ecuador & -1.3224 & $32,76 \%$ \\
\hline Colombia & -7.703 & $19,09 \%$ \\
\hline Argentina & -3.950 & $9,79 \%$ \\
\hline Perú & -2.505 & $6,21 \%$ \\
\hline Brasil & -1.940 & $4,81 \%$ \\
\hline Venezuela & -1.667 & $4,13 \%$ \\
\hline Bolivia & -1.541 & $3,82 \%$ \\
\hline Total (todos los países) & -40.361 & $100,00 \%$ \\
\hline
\end{tabular}

Fuente: elaboración propia a partir de Estadísticas de Educación.

11 De esas 67 nacionalidades, 2 son identificadas como agrupación de países.

12 En este caso de esas 72 nacionalidades, 7 son identificadas como agrupación de países. 
De cada 10 escolares que se "perdieron» del curso 2011/12 al curso 2012/13, 8 eran ecuatorianos, colombianos, argentinos, peruanos, brasileños, venezolanos o bolivianos. Si sólo tuviéramos en cuenta los descensos de estos países descubriríamos que los "perdidos» son poco más de 1 de cada 10 del conjunto. Pero debe saberse que estas siete nacionalidades suponen uno de cada cuatro $(26,62 \%)$ del total de los escolares de nacionalidad extranjera. Tales disminuciones, por lo tanto, son muy recientes y muy reducidas. Además estos datos contradicen las palabras sobre la pérdida «significativa» de escolares extranjeros. Muy probablemente lo que quieren decir es que «ya no llegan tantos como antes», pero el descenso de estos escolares no es generalizado, tampoco lo es en todo el sistema educativo y se explican por la reducción de algunas nacionalidades.

\section{INMIGRACIÓN Y EMIGRACIÓN DE LOS MENORES EXTRANJEROS EN ESPAÑA}

Estudiemos ahora este fenómeno con algún instrumento más preciso que permita saber las variaciones anuales entre los que «llegan» y los que se «marchan». Nos serviremos de los datos que ofrece la Estadística de Migraciones ${ }^{13}$ y el Padrón Municipal de Habitantes, y sólo para el caso de los menores de 14 o 16 años ${ }^{14}$.

13 Los detalles de esta Estadística se pueden consultar en la nota metodología del propio Instituto Nacional de Estadística: http://www.ine.es/metodologia/ t20/t2030277.pdf. Pero debemos advertir de la novedad de esta fuente y con ello de su proceso de elaboración complejo y no siempre «concluido». Obsérvese lo que en la nota metodológica se dice: «la propia naturaleza y finalidad administrativa del Padrón hace necesario el tratamiento estadístico de la información que en él se registra con el fin de alcanzar la mejor aproximación estadística al fenómeno. La observación de variaciones en el Padrón pudiera ser insuficiente para la medición de los flujos migratorios. Así, esta operación nace con la vocación de ir incorporando en su metodología de cálculo cuantas fuentes adicionales de información y mejoras metodológicas se consideren oportunas para una mejor identificación y medición del fenómeno migratorio».

14 La Estadísticas de Migraciones ofrece datos de edad agrupados quinquenalmente y por ello utilizaremos los tres grupos más próximos a la edad escolar obligatoria. En el caso del Padrón se ofrecen datos año a año y en ese caso construiremos el grupo 0-16 años. Obviamente no podremos comparar estos datos con los de más arriba, pero si podremos observar las tendencias de unos y otros. 
Como puede observarse en el Gráfico 4, la tendencia es a decrecer en los cinco años de la serie para el caso de la inmigración. Es decir, ya no «llega» tanta población de nacionalidad extranjera de 0 a 14 años como en años anteriores y aunque el mayor descenso se produce del año 2008 al 2009, el descenso, más moderado, continua hasta fechas más recientes.

GRÁFICO 4

EVOLUCIÓN DE LA TASA DE INMIGRACIÓN Y EMIGRACIÓN DE LA POBLACIÓN DE NACIONALIDAD EXTRANJERA (0-14 AÑOS)

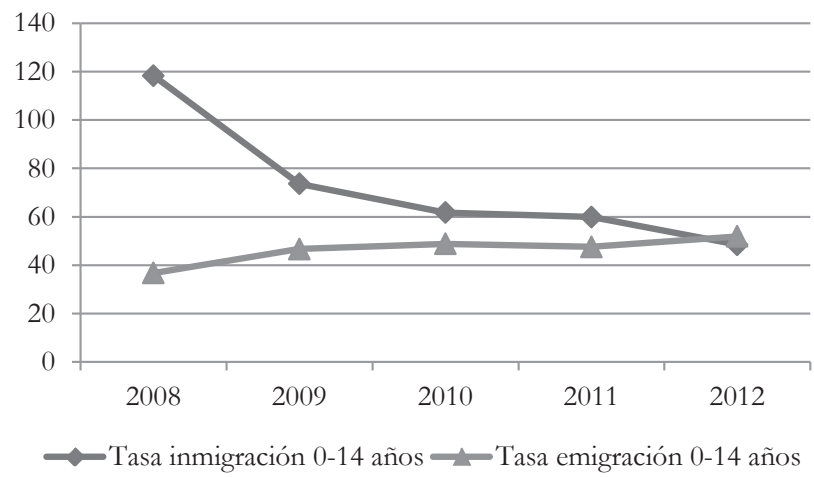

Fuente: elaboración propia a partir de los datos de la Estadística de Migraciones.

Sin embargo, aunque hablamos de descenso y de decrecimiento en todos los casos, debe entenderse que estas cifras nos indican que seguía llegando población de nacionalidad extranjera entre 0 a 14 a España. Como puede intuirse, una parte de ella acabaría en el sistema educativo. La Tabla 2 nos muestra lo que queremos decir. Los datos nos indican que el periodo analizado ha supuesto un incremento importante con una tasa bruta de inmigración que oscila entre $118,3 \%$ en el año 2008 al 48,4\%o en el año 2012. Solo en el año 2011 y el 2012 tal tasa de inmigración se ha igualado a la del conjunto de la población de nacionalidad extranjera, pero en los tres años anteriores la diferencia era notable. 


\section{TABLA 2}

EVOLUCIÓN DE LA POBLACIÓN DE NACIONALIDAD EXTRANJERA DE 0-14 AÑOS Y TOTAL EMPADRONADA Y DATOS MIGRATORIOS

\begin{tabular}{|c|c|c|c|c|c|c|c|c|c|c|}
\hline & \multicolumn{2}{|c|}{2008} & \multicolumn{2}{|c|}{2009} & \multicolumn{2}{|c|}{2010} & \multicolumn{2}{|c|}{2011} & \multicolumn{2}{|c|}{2012} \\
\hline & $0-14$ & Total & $0-14$ & Total & $0-14$ & Total & $0-14$ & Total & $0-14$ & Total \\
\hline Población & 744.359 & 526.8762 & 806.942 & 5.648 .671 & 832.666 & 5.747 .734 & 837.043 & 5.751 .487 & 833.056 & 5.736 .258 \\
\hline Inmigración & 88.039 & 567.373 & 59.414 & 365.367 & 51.412 & 330.286 & 5.0105 & 335.893 & 40.346 & 272.489 \\
\hline Tasa inmigra. & 118,3 & 107,69 & 73,6 & 64,68 & 61,7 & 57,46 & 59,9 & 58,40 & 48,4 & 47,50 \\
\hline Emigración & 27.386 & 254.927 & 37.655 & 344.128 & 40.666 & 363.222 & 3.9801 & 353.562 & 43.254 & 389.339 \\
\hline Tasa emigra. & 36,8 & 48,38 & 46,7 & 60,92 & 48,8 & 63,19 & 47,5 & 61,47 & 51,9 & 67,87 \\
\hline Saldo migra. & 60.653 & 312.446 & 21.759 & 21.239 & 10746 & -32936 & 10.304 & -17669 & -2908 & -116.850 \\
\hline Tasa migra. & 81,5 & 59,30 & 27,0 & 5.648 .671 & 12,9 & $-5,73$ & 12,3 & $-3,07$ & $-3,5$ & $-20,37$ \\
\hline
\end{tabular}

Fuente: elaboración propia a partir de los datos de la Estadística de Migraciones y Padrón Municipal de Habitantes.

El mismo Gráfico 4, citado con anterioridad, nos muestra la evolución de la emigración hacia el exterior de la población de nacionalidad extranjera que estamos estudiando. Como podemos ver, la tasa de emigración ha crecido hasta llegar a igualarse y superar la tasa de inmigración. Pero comparando dichas tasas (Ver Tabla 2) las diferencias han oscilado entre 11 a 15 puntos en los años estudiados y ello nos muestra que la emigración en el conjunto de población de nacionalidad extranjera está siendo más importante que en el subgrupo de 0-14 años. Es decir, no podemos negar que se «marchan» o «desaparecen» de las estadística, pero no parece que lo hagan en igual volumen los mayores que los menores. Estas diferencias entre el conjunto de población extranjera y el subgrupo de 0-14 años se puede observar en el Gráfico 5. 
GRÁFICO 5

TASA BRUTA DE MIGRACIÓN PARA LA POBLACIÓN DE NACIONALIDAD EXTRANJERA: POBLACIÓN Y 0-14 AÑOS

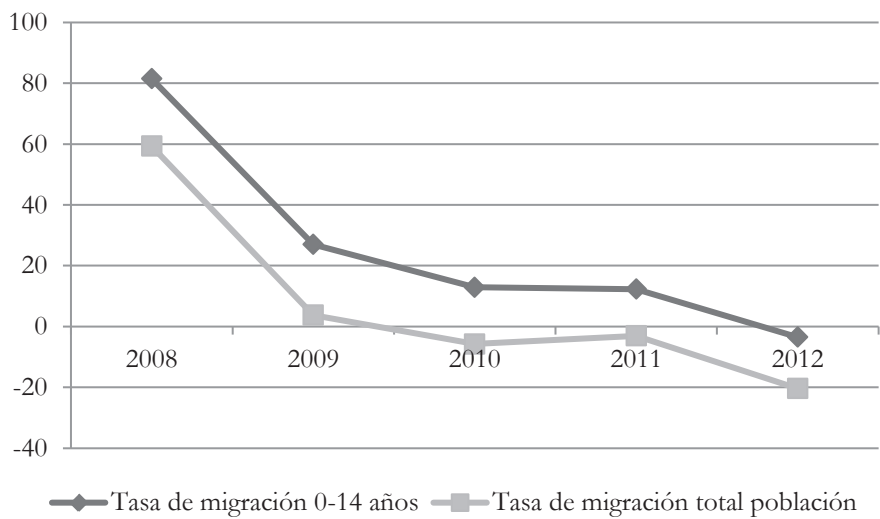

Fuente: elaboración propia a partir de los datos de la Estadística de Migraciones.

Todos los años se da una diferencia entre 15-20 puntos a favor de una mayor tasa del grupo de menores. Es decir, su saldo migratorio ha sido siempre positivo, salvo en el último año (2012), frente a la de la población en su conjunto que empezó a ser negativo en los tres últimos años. En los cinco años estudiados nos encontramos que el promedio de tasa de inmigración para toda la población de nacionalidad extranjera ha sido de $67 \%$ frente al promedio del grupo de 0-14 años que ha sido de $72 \%$. La tasa promedio de emigración para toda la población ha sido de $60 \%$ (muy próxima a la de inmigración) y para el grupo de 0-14 años ha sido de 46\%o (mucho más lejana de la tasa de inmigración del grupo). Más aún, la tasa de migración resultado del saldo migratorio presenta un dato de $6 \%$ para el caso de la población de nacionalidad extranjera y de $26 \%$ para el del subgrupo de 0-14 años. Las diferencias parecen claras y concluyentes.

Con estos primeros datos podemos afirmar que se han producido salidas de la población extranjera en edad escolar (al menos del grupo de 0-14 años), pero también se han producido entradas. En el periodo de cinco años analizado comprobamos que el total de nuevas 
incorporaciones suman 289.316 menores de nacionales extranjeros. Las salidas del Padrón suponen un total de 118.762 menores menos, lo que implica un saldo a favor de los que se incorporaron de 100.554 menores más.

Observemos estos datos desagregándolos por nacionalidad. A excepción de ocho nacionalidades (alemana, cubana, estadounidense, hondureña, rusa, ucraniana y venezolana), otras dieciocho coincidían en las lista de inmigración y emigración, si seleccionamos sólo aquellas que suman el $80 \%$ del total de los movimientos migratorios, en uno u otro sentido. Todo ello para el grupo 0-14 años.

Como podemos observar en la Tabla 3, la tasa promedio más alta de la serie estudiada es la de la nacionalidad china $(87,84 \%$ ), que supera la media $(72,39 \%$ ) del conjunto de la población de nacionalidad extranjera inmigrante de 0-14 años (ver Tabla 2). Las otras tres nacionalidades se sitúan por debajo de este promedio: rumana $(61,17 \%)$, ecuatoriana $(54,70 \%)$ y marroquí $(42,23 \%)$. Ello viene a indicarnos que aunque las cuatro nacionalidades seguían aportando nueva población inmigrante al grupo de 0-14 años, el de la nacionalidad china ha sido relativamente más importante. 
TABLA 3

EVOLUCIÓN DE LA POBLACIÓN DE NACIONALIDAD EXTRANJERA (0-14 AÑOS) EN EMPADRONADA Y DATOS MIGRATORIOS DE CUATRO NACIONALIDADES

\begin{tabular}{|c|c|c|c|c|c|c|}
\hline & & 2008 & 2009 & 2010 & 2011 & 2012 \\
\hline \multirow{7}{*}{ Rumana } & Población & 101.265 & 116.641 & 124.990 & 132.712 & 139.243 \\
\hline & Inmigración & 10.051 & 6.407 & 7.494 & 7.682 & 4.709 \\
\hline & Tasa inmigración & 99,3 & 54,9 & 60,0 & 57,9 & 33,8 \\
\hline & Emigración & 3.256 & 5.494 & 6.117 & 5.582 & 8.764 \\
\hline & Tasa emigración & 32,2 & 47,1 & 48,9 & 42,1 & 62,9 \\
\hline & Saldo migratorio & 6.795 & 913 & 1.377 & 2.100 & -4.055 \\
\hline & Tasa migratoria & 67,1 & 7,8 & 11,0 & 15,8 & $-29,1$ \\
\hline \multirow{7}{*}{ Marroquí } & Población & 139.241 & 160.072 & 179.216 & 191.721 & 199.612 \\
\hline & Inmigración & 12.484 & 8.699 & 5.364 & 4.710 & 3.520 \\
\hline & Tasa inmigración & 89,7 & 54,3 & 29,9 & 24,6 & 17,6 \\
\hline & Emigración & 2.430 & 3.257 & 3.672 & 4.466 & 4.901 \\
\hline & Tasa emigración & 17,5 & 20,3 & 20,5 & 23,3 & 24,6 \\
\hline & Saldo migratorio & 10.054 & 5.442 & 1.692 & 244 & -1.381 \\
\hline & Tasa migratoria & 72,2 & 34,0 & 9,4 & 1,3 & $-6,9$ \\
\hline \multirow{7}{*}{ China } & Población & 23.280 & 28.773 & 32.480 & 35.141 & 38.057 \\
\hline & Inmigración & 4.067 & 2.388 & 2.333 & 2.159 & 1.836 \\
\hline & Tasa inmigración & 174,7 & 83,0 & 71,8 & 61,4 & 48,2 \\
\hline & Emigración & 1.562 & 1.966 & 2.260 & 2.571 & 2.649 \\
\hline & Tasa emigración & 67,1 & 68,3 & 69,6 & 73,2 & 69,6 \\
\hline & Saldo migratorio & 2.505 & 422 & 73 & -412 & -813 \\
\hline & Tasa migratoria & 107,6 & 14,7 & 2,2 & $-11,7$ & $-21,4$ \\
\hline \multirow{7}{*}{ Ecuatoriana } & Población & 78.185 & 75.487 & 66.692 & 56.713 & 46.242 \\
\hline & Inmigración & 10.274 & 4.378 & 1.895 & 1.627 & 1.248 \\
\hline & Tasa inmigración & 131,4 & 58,0 & 28,4 & 28,7 & 27,0 \\
\hline & Emigración & 1.981 & 2.636 & 2.867 & 3.027 & 2.966 \\
\hline & Tasa emigración & 25,3 & 34,9 & 43,0 & 53,4 & 64,1 \\
\hline & Saldo migratorio & 8.293 & 1.742 & -972 & -1.400 & -1.718 \\
\hline & Tasa migratoria & 106,1 & 23,1 & $-14,6$ & $-24,7$ & $-37,2$ \\
\hline
\end{tabular}

Fuente: elaboración propia a partir de los datos de la Estadística de Migraciones y Padrón Municipal de Habitantes. 
En lo relativo a la emigración la tasa media de este grupo etario fue en la serie estudiada de 46,35\%o (26 puntos menos que la tasa de inmigración) y de nuevo la nacionalidad china $(69,55 \%$ ) es la que presenta una tasa más alta. Es decir, igual que entraban muchas personas de dicha nacionalidad también salían muchas. Por su parte, se sitúan en datos similares a la media de todas las nacionalidades la rumana $(46,64 \%$ ) y ecuatoriana $(44,15 \%$ ) y muy por debajo la nacionalidad marroquí $(2,23 \%)$. Es decir, esta última nacionalidad era la que menos emigración registraba en términos relativos.

Por último, la tasa migratoria media de la serie analizada presentaba para el conjunto de la población de ese grupo de edad un dato de $26,03 \%$, lo que viene a decirnos que el resultado era positivo a favor de la inmigración. Las cuatro nacionalidades analizadas presentaban en este caso también tasas positivas de migración, pero más bajas que el valor promedio del conjunto. La nacionalidad marroquí $(22 \% 0)$ era la que más se acercaba a la media, seguida de la china $(18,29 \%)$, la rumana $(14,53 \%)$ y la ecuatoriana $(10,55 \%)$. Siendo, como se puede ver, en esta última donde estaba empezando a tener una incidencia importante el fenómeno emigratorio, bien de retorno o bien de inicio de una emigración a otro país.

\section{3. ¿REALMENTE ESTAMOS HABLANDO DE RETORNO DE MIGRANTES?}

Ya indicábamos al principio de nuestro texto que las fuentes disponibles para el estudio del retorno de los migrantes no gozan de muy buena salud. Tienen ya más de dos décadas las palabras de José Castillo cuando nos decía que carecemos de un "conocimiento teórico del regreso de emigrantes» y que «... la sociología de la migración de retorno... adolece de deficiencias teóricas» (Castillo Castillo, 1997: 30-31). Más tarde sería Jorge Durand quien veía cómo las migraciones de retorno no habían corrido la misma suerte en la producción de teorías que las analizaran como las migraciones en general:

Las diversas teorías que explican el fenómeno migratorio internacional han demostrado, en la práctica, ser acercamientos parciales a una realidad compleja y cambiante... las teorías 
vigentes han atendido de manera tangencial el corolario del fenómeno: el retorno, que si bien no es un fenómeno generalizado y suele ser selectivo, en muchos casos resulta relevante (Durand, 2006: 167-168).

La propia conceptualización del término «retorno» y las varias tipologías de migración que engloba es, sin duda, una de las causas de ausencia de producción teórica. Otro gran clásico del estudio del retorno, Bovenkerk (1974), propuso la necesaria clarificación conceptual con los siguientes términos: «migración de retorno», «migración de tránsito», «reemigración», «nueva emigración», «migración circular». Más recientemente será el ya citado Duran (2006) quien nos haga otra propuesta en este sentido: «retorno voluntario del migrante establecido», "retorno del migrante temporal», «retorno transgeneracional», «retorno forzado», «retorno del fracasado» y «retorno programado». Y Domingo y Sabater terminan por aclararnos lo que puede haber bajo el término de «retorno»: «retorno propiamente dicho», "reemigración» y «migraciones de arrastre» (Domingo y Sabater, 2013: 67).

En fin, falta de concreción y definición de lo que se pretende estudiar que ha motivado en los últimos tiempos tanto las revisiones detalladas de lo que se ha realizado en este terreno como las proposiciones teóricas más elaboradas. Para el primer asunto basta citar los trabajos más recientes de revisión bibliográfica de Fernández (2011) y de Tovar y Victoria (2013). Para el caso de las propuestas teóricas contamos con un trabajo de reconocimiento internacional donde se analiza cómo las teorías migratorias han tratado el retorno de los migrantes (Cassarino, 2004) y con propuestas muy recientes ligadas a los estudios trasnacionales que nos advierten de la necesidad de tratar el retorno como una forma de migración más que «sólo puede ser entendido desde una perspectiva que lo comprenda inmerso en la dinámica de las relaciones, prácticas y experiencias en las que se entrelazan las sociedades de origen y de asentamiento» (Cavalcanti y Parella, 2013: 16).

Otro aspecto que nos gustaría señalar aquí es que si algo sabemos sobre el retorno es que además de las aproximaciones estadísticas que hemos tratado de presentar en los anteriores apartados, los estudios micro sobre el terreno son imprescindibles para entender bien tal movilidad. Lo que hemos presentado nos muestra que ha existido y existe alguna forma de retorno. Ello ocurría incluso 
cuando la inmigración era muy importante y se sigue produciendo ahora. Aunque los datos mostrados de diferentes fuentes utilizadas no son comparables, si lo son las tendencias. Comentemos algo sobre éstas:

- En el sistema educativo observamos que se ha producido en el último curso analizado la primera reducción absoluta en la presencia de población de nacionalidad extranjera escolarizada. Es decir, que son menos los escolares de este grupo respecto del curso anterior. Tales pérdidas supusieron un descenso respecto del año anterior de algo menos del 3\%. Y todo ello sin descontar las «bajas» de escolares de nacionalidad extranjera motivadas por la obtención de la nacionalidad española, de las que no deberíamos hablar en términos de retorno sin disponer de más datos ${ }^{15}$. No son datos que nos hablen directamente del retorno, indican «pérdidas» de escolares de nacional extranjera.

- En el caso de los datos de la Estadística de Migraciones hemos observado que también es al final de los años analizados cuando se produce un descenso en forma de saldo migratorio negativo, pero tal saldo se sitúa en -2.908 menores menos. Una cantidad que representa en ese grupo etario un $0,35 \%$. Es decir, existe una tendencia a la reducción, pero reciente y pequeña.

¿Cómo podemos explicar entonces que el discurso mediático y el discurso de los responsables públicos haya generado esa asociación tan lineal entre «retorno» de los que fueron inmigrantes y «crisis económica»? Una de las razones ya la anunciamos al principio de este texto, el deseo del retorno asociado a toda migración. Pero también dijimos que se trataba de una explicación simplista y carente de argumentos empíricos. Lo que si hemos mostrado aquí es que la reducción de población extranjera en el sistema educativo no nos permite hablar de retorno importante ni de una «desaparición».

15 Recordemos lo que apuntan Andreu Domingo y Albert Sabater sobre lo que pueden suponer las nacionalizaciones en relación con la movilidad de esta población en general: «En este contexto debe subrayarse que la adquisición de la nacionalidad española por parte de un número significativo de la población extranjera (551.162 entre el año 2002 y 2010) podría desencadenar una mayor proporción de migrantes transitorios, o sea, aquéllos que exhiben una elevada propensión a moverse circularmente y más allá entre dos o más países, principalmente teniendo en cuenta el espacio de movilidad europeo que permiten los acuerdos de Schengen de 1996» (Domingo y Sabater, 2013: 84). 
Veamos otras causas para explicar los discursos que afirman que «se están marchando...».

Durante la primera década del siglo y coincidiendo con la llegada importante de población extranjera, el sistema educativo desarrolló diferentes iniciativas para atender a esta nueva población. Todo el mundo coincide en que aquéllas que se han dedicado a la atención de los nuevos escolares para acercarles a la lengua vehicular de la escuela han sido las más importantes. De esta manera, han surgido en cada Comunidad Autónoma diferentes tipos de aulas para «acoger» a estos escolares y enseñarles la lengua de la escuela. En Andalucía se han llamado Aulas Temporales de Adaptación Lingüística $(\mathrm{ATAL})^{16}$ y en cada Comunidad reciben un nombre distinto ${ }^{17}$.

Nuestro objetivo en las siguientes páginas es, comprobar en qué medida estos recursos se han visto disminuidos fruto de la mayor o menor presencia de estos escolares y para ello haremos una comparación entre los efectivos de estos recursos y el número de estos escolares.

Hemos tomado dos casos concretos (ver Tabla 4). Por un lado, los datos corresponden al sistema educativo de Andalucía y a su población de nacionalidad extranjera, y por otro, los datos de la Comunidad de Madrid ${ }^{18}$. Para el caso de Andalucía las ATAL se han visto reducidas de manera importante desde el curso 2009/10. El curso anterior se alcanzó la cifra de 310 ATAL para terminar en el curso 2012/13 con 66 aulas menos. Desde ese curso de 2009/10 hasta el último estudiado el valor promedio de reducción ha sido de -5,78.

16 Las ATAL son un recurso educativo de la Consejería de Educación de la Junta de Andalucía, gestionado por sus Delegaciones Provinciales, cuyo profesorado forma parte de la Educación Compensatoria. Aparecen por primera vez en el Plan para la Atención Educativa del Alumnado Inmigrante en 2001 con el objetivo de «Potenciar programas de apoyo para el aprendizaje de la lengua española», aunque comenzaron el curso 1997-98 en Almería. La norma "oficial» de su funcionamiento es de 2007.

17 La denominaciones de estas aulas son muy diversas: Aulas de Acogida en Cataluña, Programa de Acogida Lingüística y Cultural (PALIC) en Baleares, Aulas de Adaptación Lingüística y Social (ALISO) en Castilla-León, las Aulas de Enlace en Madrid, etc.

18 La elección tiene que ver con el signo ideológico diferente de los gobiernos en ambas Comunidades. En Madrid ha gobernando el Partido Popular, conservador, y en Andalucía el Partido Socialista Obrero Español, progresista. En cada caso se ha elaborado un discurso sobre las medidas que se deberían desarrollar en época de crisis para con los servicios públicos. 
El alumnado de nacionalidad extranjera ${ }^{19}$ también se ha visto reducido en la serie estudiada, pero en mucha menor medida. Llegó a alcanzar la cifra de 63.635 en enseñanzas de primaria y secundaria obligatoria, para terminar la serie estudiada con 58.240. El promedio de reducciones interanuales desde que comenzó el descenso es de $-2,19 \%$, sensiblemente menor a la reducción observada en los efectivos de estas «aulas especiales». Las tendencias son similares si comparamos las reducciones de la población empadronada de nacionalidad extranjera (6-16 años $)^{20}$ : un promedio de aumento de 3,84 .

19 Se debe tener en cuenta que esta «aulas especiales» no son para todo el alumnado de nacionalidad extranjera, sino para aquellos que carezcan de competencia en la lengua vehicular de la escuela o su competencia lingüística sea muy reducida. No obstante, nuestras investigaciones han mostrado que estos recursos han terminado siendo "refugio" de otros escolares de nacionalidad extranjera, independiente del nivel de competencia lingüística.

20 Estas «aulas especiales» solo se encuentran implantadas en educación primera y secundaria obligatoria y las edades «normales» para tales enseñanzas es de 6-16 años. 
TABLA 4

EVOLUCIÓN DE «AULAS ESPECIALES» PARA POBLACIÓN DE NACIONALIDAD EXTRANJERA EN ANDALUCÍA Y EN MADRID Y EVOLUCIÓN DEL ALUMNO DE NACIONALIDAD EXTRANJERA (PRIMARIA Y SECUNDARIA) Y POBLACIÓN EMPADRONADA DE NACIONALIDAD EXTRANJERA (6-16 AÑOS) ${ }^{21}$

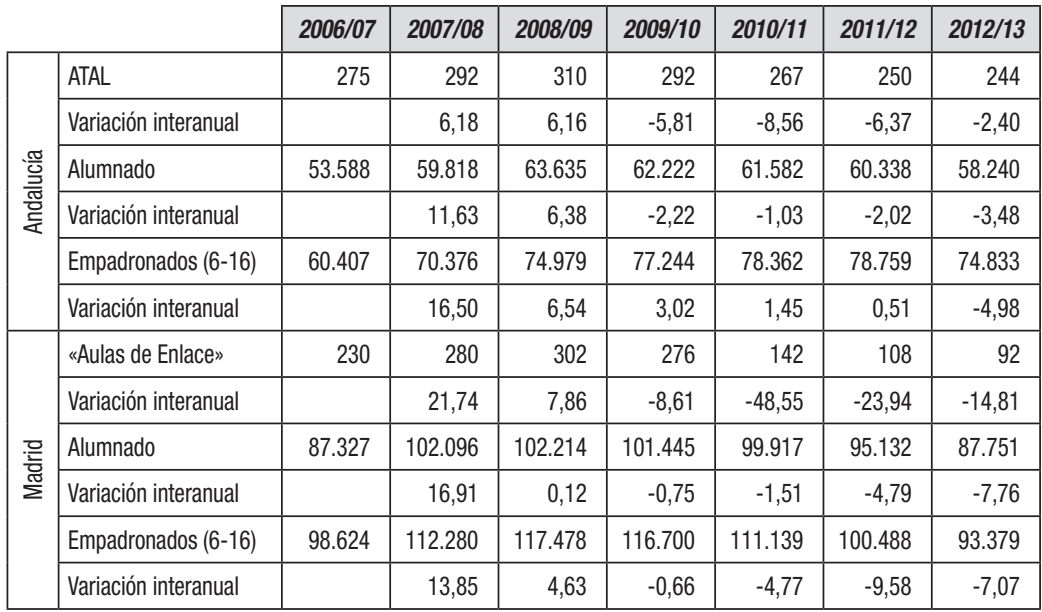

Fuente: elaboración propia a partir de Dirección General de Participación e Innovación Educativa (ATAL), Consejería de Educación y Empleo de la Comunidad de Madrid («Aulas de Enlace»), Estadísticas de Educación (escolarizados de nacionalidad extranjera) y Padrón Municipal de Habitantes (empadronados 6-16 años de nacionalidad extranjera)

La reducción del último año estudiado en las ATAL fue menor porcentualmente que la reducción ocurrida en la población escolar y en la población empadronada, pero es que los ajustes ya se hicieron con anterioridad. En los cursos 2009/10, 2010/11 y 2011/12 se reducían las ATAL en un $-5,81 \%,-8,56 \%,-6,37 \%$ respectivamente, cuando los escolares solo se reducían en un $-2,22 \%,-1,03 \%$ y-2,02\%

21 Los datos escolares se presentan por cursos escolares y los datos demográficos por años naturales. Los datos escolares son del mes de septiembre del año que inicia la cifra del curso escolar y los datos demográficos del Padrón Municipal de Habitantes (1 de enero de cada año) son los que termina la cifra del curso escolar. Es decir, bajo la columna del curso 2006/07 los datos escolares que aparecen son de septiembre de 2006 y los datos de demográficos son de enero de 2007. 
respectivamente, o los menores empadronados no presentaban tasas de reducción interanual $(3,02 \%, 1,45 \%$ y $0,51 \%$ respectivamente).

Pasemos a mirar los datos con mayor detalle. En el curso 2007/08, para un total de 59.818 escolares (muy similar la cifra a los 58.240 escolares del curso 2012/13) se disponía 292 ATAL, lejos de los 244 del curso 2012/13 (48 aulas menos). En el 2009 los menores de nacionalidad extranjera (6-16 años) que se encontraban empadronados eran 74.979, en el 2013 eran 74.833 (cifras muy similares). En 2009 las ATAL eran 310 y en 2013 eran 244 (66 aulas menos).

Veamos ahora el caso de la Comunidad de Madrid. En esta Comunidad la reducción de las «Aulas de Enlace» comenzó también en el curso 2009/10. Desde entonces, las reducciones han supuesto un promedio del $-23,98 \%$. Los cambio en los escolares de nacionalidad extranjera y los menores (6-16 años) empadronados de nacionalidad extranjera también han existido. El promedio para estos grupos en las mismas fechas ha sido del 3,70\% (escolares) y del -0,60\% (empadronados), es decir, los escolares no tiene un promedio de decrecimiento en el periodo estudiado, aunque sí los empadronados de 6 a 16 años. Mientras que las «Aulas de Enlace» han pasado de ser un tercio de lo que eran en el curso 2008/09 (han pasado de 302 a 92) los efectivos de población se han visto reducir entre un $10 \%$ y un $15 \%$. Aquí las diferencias son mucho más notables que en Andalucía. Para 87.327 escolares de nacionalidad extranjera se disponía de 239 «Aulas de enlace» en el curo 2006/07 y para 87.751 escolares eran en el curso 2012/13 tan sólo 93 aulas (138 aulas menos). No creemos que sea necesario insistir sobre unas diferencias que son tan evidentes ${ }^{22}$. Y aunque alguien pudiera argumentar que la presencia de población de nacionalidad extranjera en la escuela no precisa ya de tales recursos, pues se encuentra integrada por haberse incorporado hace varios cursos escolares, no debe perderse de vista que las cifras de escolares de nacionalidad extranjera y las de menores empadronados de nacionalidad extranjera nos permiten afirmar que el fenómeno de la inmigración persiste. Aunque desciendan los datos tipo stock y los efectivos de esta población, una parte de los que se contabilizan son población «nueva», son «nuevos inmigrantes», y no nos referimos a los nacimientos de la población de nacionalidad

22 Para el caso de la Comunidad de Madrid estas diferencias entre poblaciones y recursos ha sido descrita detalladamente por García Fernández y Moreno Herrero (2014). 
extranjera. Aunque la emigración aumente, la inmigración no se ha parado. Aunque aquélla ya sea más importante que ésta, la inmigración no ha desaparecido.

Volviendo al grupo 5-14 años y los datos que nos ofrecía la Estadística de Migraciones, debemos indicar que en los años 2008, 2009, 2010, 2011 y 2012, los efectivos de nuevas entradas (inmigrantes) fueron respectivamente los siguientes: 63.355, 41.002, 33.947, $33.128,26.564$. De ninguna manera podemos admitir que hayan desaparecido estos flujos migratorios de entrada del extranjero.

Podemos observar algún tipo de movilidad en la población de menores de nacionalidad extranjera y es muy posible que una parte de esa movilidad sea de retorno a sus países, pero no parece fácil que podamos justificar las reducciones de los recursos dedicados a estas poblaciones en la escuela por su reducción como grupo. Todo indica que las autoridades están observando que en situación de dificultades económicas para una buena parte de la población, mantener los recursos de apoyo a la población extranjera podría ser de difícil justificación para la población autóctona y han optado por su reducción. No es otra cosa que poner en práctica el xenófobo lema —vociferado por algunos partidos políticos- «primero para los de aquí...»

\section{BIBLIOGRAFÍA}

BovenKerk, F. (1974): The Sociology of Return Migration. La Haya, Martinus Nifhoff.

CASSARINO, J. P. (2004): «Theoris in greturn migration: The conceptual approach to return migrants revisited», International Journal on Multicultural Societies, 6 (2), pp. 253-279.

Castillo Castillo, J. (1997): «Teorías de la migración de retorno», en Izouierdo Escribano, A. y Álvarez Silvar, G. (coord.), Políticas de retorno de emigrantes. A Coruña, Univeridade da Coruña, pp. 29-44.

Cavalcanti, L. y Parella Rubio, S. (2013): «El retorno desde una perspectiva trasnacional». REMHU-Revista Interdisciplinar da Mobilidade Humana, XXI, 41, pp. 9-20.

Colectivo IOE (2012): Impacto de la crisis sobre la población inmigrante. Madrid, Organización Internacional para las Migraciones.

Domingo I Valls, A. y Recaño Valverde, J. (2010): «La inflexión en el ciclo migratorio internacional en España Impacto y consecuencias 
demográficas», en AJA, E.; Arango, J. y Oliver, J. (eds.), La inmigración en tiempos de crisis. Anuario de la inmigración en España (edición 2009). Barcelona, CIDOB, pp. 182-207.

Domingo, A. y Sabater, A. (2013): «Crisis económica e inmigración: la perspectiva demográfica», en AJA, E., Arango, J. y Oliver, J. (eds.), Inmigración y crisis: entre la continuidad y el cambio. Anuario de inmigración en España. Barcelona, Centro de Estudios y Documentación Internacionales de Barcelona, pp. 60-87.

Durand, J. (2006): «Los inmigrantes también emigran: la migración de retorno como corolario del proceso». REMHU-Revista Interdisciplinar da Mobilidade Humana, xIV, 26-27, pp. 167-189.

FERnÁNDEZ GuZMÁn, E. (2011): «Revisión bibliográfica sobre la migración de retorno». Norteamérica. Revista Académica del CISAN-UNAM, 6 (1), pp. 35-68.

García Castaño, F.J.; Gijón Sánchez, M.T.; Sánchez Hitos, M.T. y Bolívar MuÑOZ, J. (2005): «Extranjeros y escolares. Formas de construir la diferencia en el ámbito de la educación formal en Andalucía mediante la llamada educación intercultural», en MuÑoz CRUz, H. (ed.), Lenguas y educación en fenómenos multiculturales. México, UAM y UPN, pp. 25-62.

García Fernández, J.A. y Moreno Herrero, I. (2014): Escuela, diversidad cultural e inclusión. Madrid, La Catarata.

López De Lera, D. (2010): «Emigración, inmigración y retorno: tres etapas de un mismo proceso». Polígonos. Revista de Geografía, 20, pp. 9-27

Tovar Cuevas, L.M. y Victoria Paredes, M.T. (2013): «Migración internacional de retorno y emprendimiento revisión de la literatura». Revista de Economía Institucional, 15 (29), pp. 41-65. 